\title{
Modelamiento termodinámico de los inhibidores de hidratos de gas (metanol) como formadores de depositaciones inorgánicas en sistemas de hidrocarburos*
}

\author{
Carlos Iván Castillo Benavides" \\ Jhon Alexander Chiriví Tenjo ${ }^{* *}$
}

\section{Resumen}

El presente estudio modela el efecto causado al adicionar un inhibidor de hidratos de gas termodinámico como el metanol, en la formación de depositaciones inorgánicas de barita, calcita, halita, yesoy celestita. Paraello se utilizaron comofuentes bibliográficas diversos artículos publicados por la Sociedad de Ingenieros de Petróleos - SPE-y artículos publicados por investigadores de la Universidad de Rice en la SPE.

Para modelar este efecto, se realizó una herramienta de cómputo en lenguaje de programación visual basic 6.0 denominada "OilScaleInhibidor", que fue adicionada al programa "OilScale" desarrollado por el grupo de investigación de comportamiento de fases de la Universidad Surcolombiana COFA. La función de esta herramienta de cómputo, es predecir las depositaciones inorgánicas de barita, calcita, halita, yeso y celestita ocasionadas al adicionar metanol en sistemas gas/metanol/agua/sal.

La herramienta de cómputo OilScalelnhibidor, posee una exactitud aceptable en la predicción de las depositaciones inorgánicas ocasionadas por el efecto de adicionar inhibidor de hidratos de gas. Los valores obtenidos de indice de saturación mediante las diferentes fuentes analizadas generaron errores promedio absolutos de $8.5 \%$.

Palabras Clave: OilScaleInhibidor, hidratos de gas, fuentedeenergia, industria petrolera, producción de pozo

\section{Abstract}

The present studied modeled the effect caused to add an inhibitor of gas hydrates thermodynamic as methanol, in the formation of inorganic deposits barite, calcite, halita, gypsum and celestite.

"Camen Pinzón. Dinctora Proyacto de Grado. Esp. Sistemas Dinámicos. Profesora Titular. Facultad de Ingenieria. Universidad Surcolombiana carpinzonQusco.edico

** Ingenisuro de Petróleos. Universidad Surcolombiana.

*th Ingeniero de Petróleos. Unversidad Surcolombiana. 
This was used as a bibliographical sources several articles published by the Society of Petroleum Engineers SPE and articles published by researchers at Rice University in the SPE.

To model this effect was made a computational tool in programming language Visual basic 6.0 called "OilScaleInhibidor", which was added to the program "OilScale" developed by the research group behavior phases of the University Surcolombiana COFA. The function of this computational tool is predict depositaciones inorganic barite, calcite, halita, gypsum and celestite caused systems to add methanol in gas / methanol / water / salt.

The computing tool OilScaleInhibidor has an acceptable accuracy in the prediction of inorganic depositaciones caused by the effect of adding inhibitor of gas hydrates; index values obtained from saturation analyzed by the different sources generated average absolute error of $8.5 \%$.

Keys Words: OilScaleInhibidor, hydrates of gas, energy source, oil industry, well production.

\section{Introducción}

$\square$ n la actualidad es muy común hablar de hidratos de gas, debido a que se han convertido en una fuente de energía por su capacidad para almacenar compuestos como el metano, pero también son un problema en la industria petrolera porque taponan las líneas de flujo, válvulas, choques y demás accesorios que son utilizados en las facilidades de producción. Es importante, inhibir los hidratos de gas por las pérdidas que ocasionan en la producción y transporte de gas.

Para que los hidratos se formen, se deben presentar regimenes de altas presiones y bajas temperaturas. Una vez formado el hidrato de gas es necesario inhibirlo, pero al utilizar un inhibidor de hidratos de gas termodinámico éste produce un efecto adverso que es el de la formación de depositaciones de inorgánicos.

A partir de los problemas anteriormente mencionados, los investigadores han encaminado sus esfuerzos a estudiar el fenómeno de la depositación de compuestos inorgánicos originados por uso de inhibidores de hidratos de gas en sistemas gas/metanol/ agua/sal, con el objetivo de entender el comportamiento y el mecanismo de formación de estos compuestos; además de generar modelos que permitan predecir las condiciones bajo las cuales se presentaría esta depositación, pudiendo implementar operaciones de tipo preventivo, antes que de tipo correctivo.
La herramienta de computo Orl Scaleinhibidor diseńado por los autores de este trabajo, ofrece grandes ventajas, ya que permite anticiparse al problema de depositación inorgánica formada por la adición de inhibidores de hidratos de gas durante la producción del pozo, ahorrando tiempo y dinero en operaciones de reacondicionamiento de pozos.

\section{Descripción del proyecto}

\section{Hídratos de gas}

Los hidratos de gas, son compuestos conocidos desde 1811, cuando $\mathrm{H}$. Davy descubrió la existencia de hidratos de cloro y posteriormente se comprobó que otros gases también formaban este tipo de compuestos; se encuentran en diferentes partes del mundo, como se observa en la figura 1 :

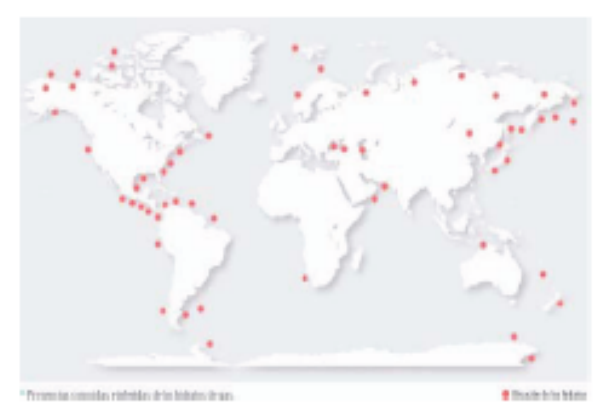

Fgura 1. Ubicación de los hidratos de gas a nivel mundial 
Los hidratos de gas se presentan como sólidos cristalinos similares al hielo, y con una composición variable según las condiciones fisicas al momento de su formación. Resultan de la combinación de moléculas de agua que se disponen en una estructura reticular de simetría cúbica que alberga entre el reticulado de moléculas un gas que puede ser $\mathrm{CO} 2$, un hidrocarburo liviano $y$ en menor medida otros gases. Genéricamente se los denomina clatratos, término que deriva del latín y significa "enjaulado". En la naturaleza, los más comunes son combinaciones de metano y agua, por lo que generalmentese toman comosinónimoslos términos hidratos demetano e hidratos de gases, pero en menor proporción pueden albergar también etano, propano y hasta butano. Como consecuencia de su composición variable, también lo son sus propiedades físicas, lo que dificulta su estudio y correcta caracterización.

\section{Estructura y manual de la herramienta de cómputo}

El programa OilScale diseríado para la predicción de depositaciones inorgánicas y ampliado para la inhibición de hidratos de gas como formadores de depositaciones inorgánicas, es un programa de fácil manejo en lenguaje de programación Visual Basic.

Al ejecutarse la herramienta de cómputo "OilScaleInhibidor" se despliega la pantalla de presentación (figura 2), donde el usuario puede conocer la aplicación del programa.

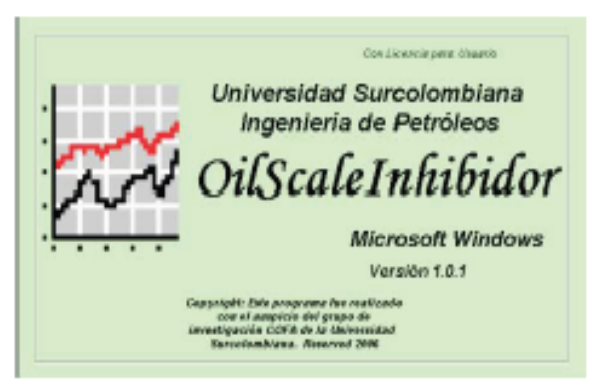

Figura 2. Presentación del programa "OilScalel nhibidor"

Luego de la presentación del programa aparece un formulario para Inicio de sesión, donde se introduce el nombre de la persona que va a ejecutar el programa, información sobre el campo y el pozo de producción de petróleo y/o gasque se vaa evaluar, a demás depresentar una casilla para escribir los comentarios referentes al pozo que se va a modelar. (Figura 3 ).

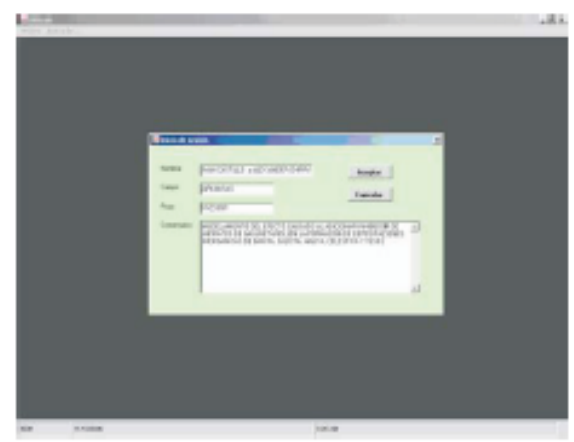

Figura 3. Inicio típico de sesión en el programa

Después de ingresar estos datos, se muestra el menú principal, donde se pueden apreciar las diferentes opciones que tiene el programa Oilscale.

El primer comando corresponde al de Archivo. donde se despliegan las opciones de nuevo, abrir o guardar un proyecto. Además tiene un ejemplo de aplicación, propiedades del proyecto y una opción para salir del programa. $\mathrm{Al}$ activar cualquiera de los comandos, nuevo o abrir, aparece el formulario para ingresar los diferentes datos necesarios para correr el programa OilScale inhibidor (figura 4) como concentración de los diferentes iones que pueden estar presentes en el fluido a analizar, caudales de aceite, agua y gas, datos de presión y temperatura de la locación evaluada, $\mathrm{pH}$ y la fracción de metanol (\% V/V) adicionada para la inhibición de hidratos de gas. Igualmente en la figura 4, aparecen los campos para mostrar densidad, sólidos totales disueltos y suma de cationes y aniones calculados por el programa. En este formulario, además aparecen los botones de calcular y salir del formulario. 


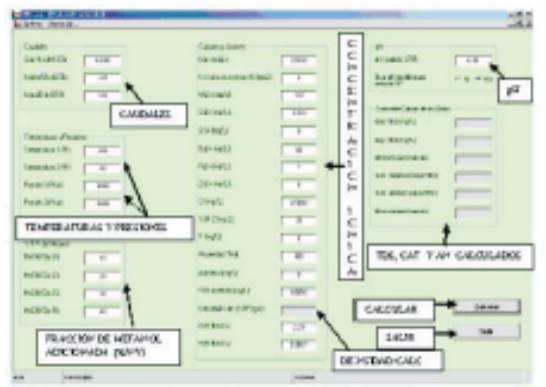

Plgura 4. Datos de entrada del programa.

El segundo comando corresponde a los Resultados del programa, donde aparecen los diferentes resultados obtenidos al ejecutar el programa. Este menú muestra cuatro opciones las cuales son predicción, inhibición en los que se pueden observar los resultados de los indices de saturación para las diferentes depositaciones que maneja el programa (barita, calcita, halita, yeso y celestita), además de una serie de gráficos que facilitan el análisis de los resultados obtenidos. La tercera opción es guardar resultados y la cuarta opción es imprimir los resultados. Una vez activado el botón Calcular, el programa realiza los cálculos pertinentes al modelo termodinámico descrito para la predicción de depositaciones inorgánicas y los cálculos del efecto causado al adicionar inhibidor de hidratos de gas (metanol) en la formación de depositaciones inorgánicas.

En este mismo formulario aparecen la densidad calculada, los sólidos totales disueltos y la suma de aniones y cationes, (figura 18). En este gráfico se puede hacer un control de los datos de entrada como lo son sólidos totales disueltos (TDS) calculados y experimentales, la suma de aniones y cationes con sus respectivas diferencias en fracción, tanto para TDS como para los iones presentes.

En la opción de índice de saturación se pueden apreciar las diferentes depositaciones que evalúa el programa tales como Calcita. Halitay Sulfatos (Barlta, Yeso y Celestita).

Al hacer clic en cualquiera de las opciones (depositaciones a predecir), aparece una tabla de resultados que muestra, las temperaturas y presiones analizadas, el indice de saturación (IS) y la masa depositada para cada una de las fracciones de metanol que fueron adicionadas para la inhibición de los hidratos de gas que fueron calculadas. Para el caso de la calcita aparece el $\mathrm{pH}$ para las presiones y temperaturas evaluadas, (figura 5).

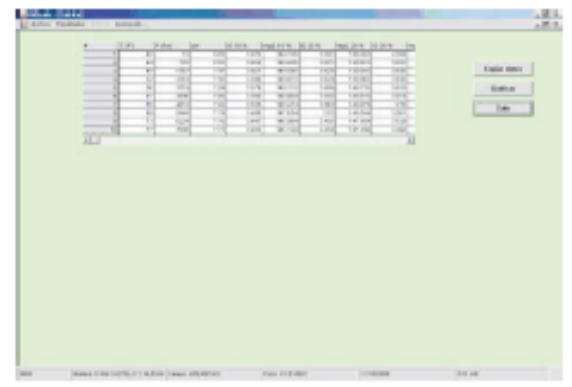

Figura 5. Tabla de resultados para la depositación de calcita

Como se puede observar en la figura 5 , aparecen los botones de copiar datos, graficar y salir. La opción Copiar Datos tiene como función copiar los datos calculados en la tabla de resultados y poder trabajar estos resultados en otro formato, por ejemplo una hoja electrónica (Excel), para ello se debe seleccionar la columna o columnas que se desean copiar y luego se da clic en copiar datos.

Laopción Graficar, tiene comofunción realizaruna gráfica de Indice de Saturación contra Temperatura dela cual se realiza el modelamiento delasposibles depositaciones inorgánicas, para este caso Calcita. Además posee cuadros de texto en los cuales se tienen los iones presentes $y$ botones de comando en donde se puede modificar la concentracion de cada uno de los iones presentes. Al igual que para la calcita, los resultados generados para las demás depositaciones inorgánicas muestran la tabla de resultados y la grafica del índice de saturación contra temperatura.

En la opción de Cambio Índice de Saturación (figura 6) se pueden apreciar la gráfica del IIS de las diferentes depositaciones que evalúa el programa contra la fracción de metanol $(\% \mathrm{~V} / \mathrm{V})$ adicionado para la inhibición de los hidratos de gas. (Figura 7). 


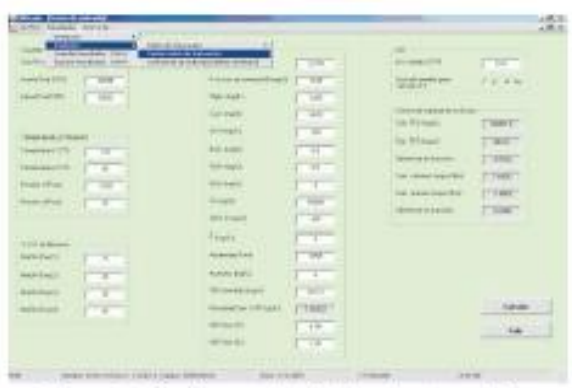

Pgura 6. Opción cambio indice de saturación.

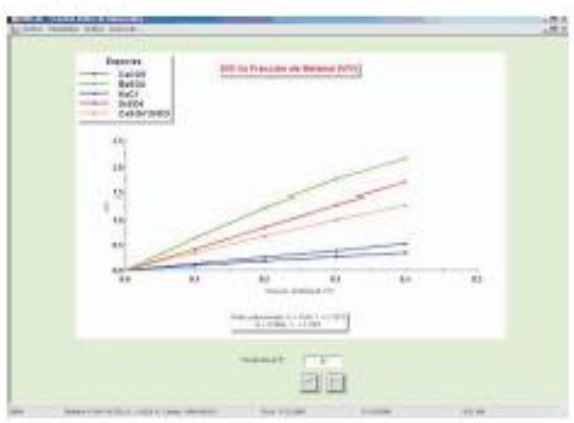

Figura 7. Gáfica $\Delta I S$ contra fracción de metanol $(92 / V)$

La opción Coeficientes de Actividad debidos al metanol (figura 8), tiene la función de mostrar una tabla de resultados, los diferentes coeficientes de actividad debidos al adicionar metanol $(\% \mathrm{~V} / \mathrm{V})$.

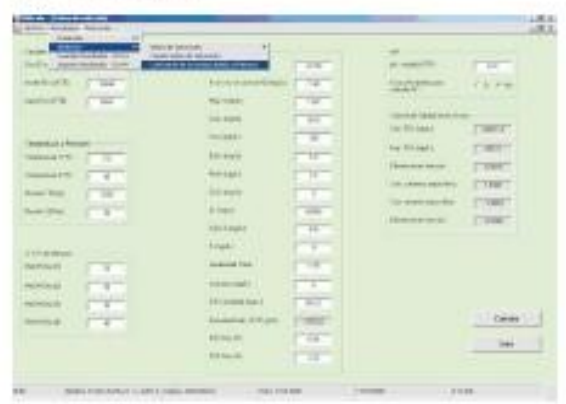

Figura 8. Opción coeficientes actividad debidos a al metand

Las diferentes gráficas generadas por el programa de cómputo poseen un menú como se muestra en la figura 9 para realizar distintas operaciones tales como, propiedades de la gráfica que es básicamente el setup de la gráfica (cambio del tipo de lineas, colores, etc.) (Figura 10). La otra opción que se tiene, es la de devolver el aumento, después de ser activado con el botón primario del mouse y encerrar el área que se quiere ampliar (figura 11). La última operación que se puede realizar con las gráficas, es la de guardarla como formato de mapa de bits (.bmp), saliendo un cuadro de diálogo para digitar el nombre que se desee para la gráfica y la dirección en la cual quiera guardarla.

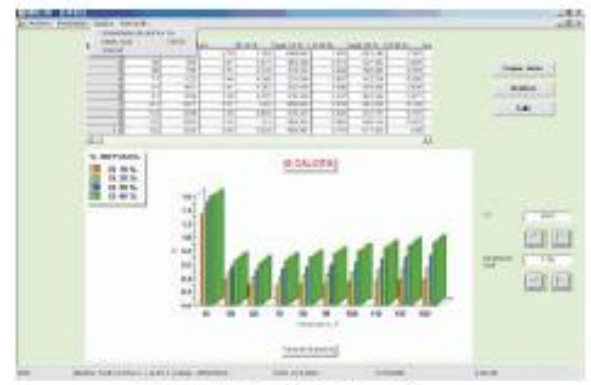

Figura 9. Menú de las gráficas

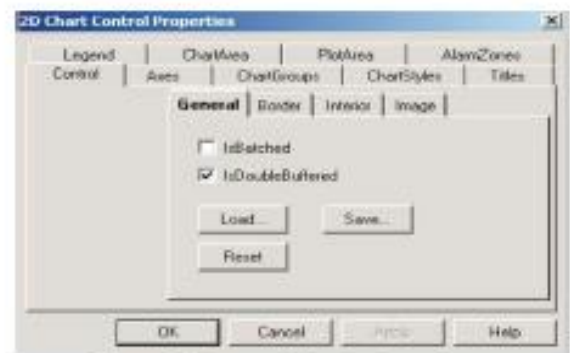

Figura 10. Propiedades de las gáficas

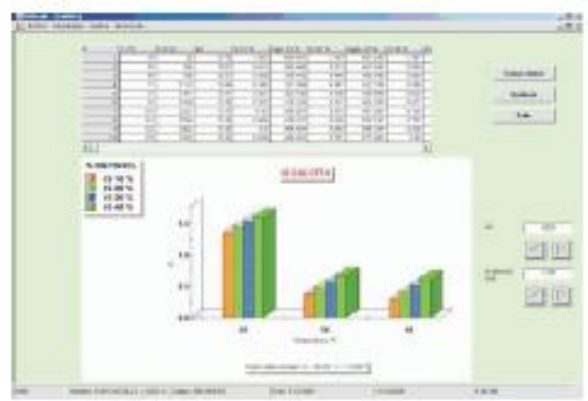

Fgura 11. Gráfica con aumento

Después de realizado el respectivo modelamiento, en el menú de resultados se encuentra la opción 
de guardar los resultadoso imprimirlos (figura 12); si los resultados se quieren guardar estos quedan en un formato de texto (.txt), como es mostrado en la figura 13.

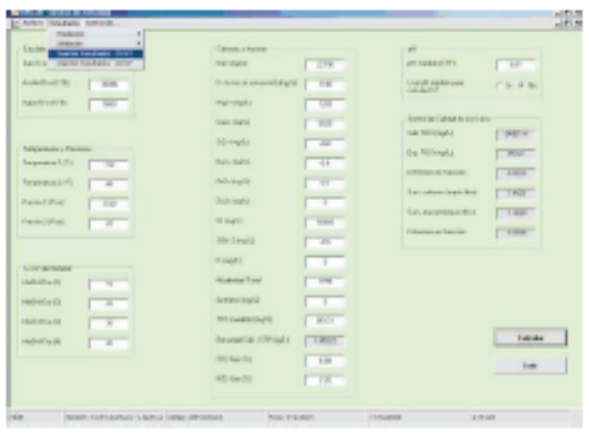

Figura 12. Opción guardar resultados

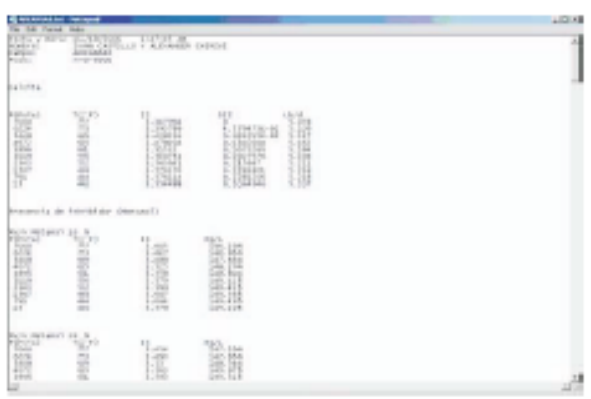

Figura 13. Resultados guardados en formato .txt.

De acuerdo con la figura 4 , el último comando de este formulario y por ende del programa, es el del menú "Acerca de..", donde se muestra la descripción del programa, los diseríadores del mismo y la información básica del sistema (figura 14).

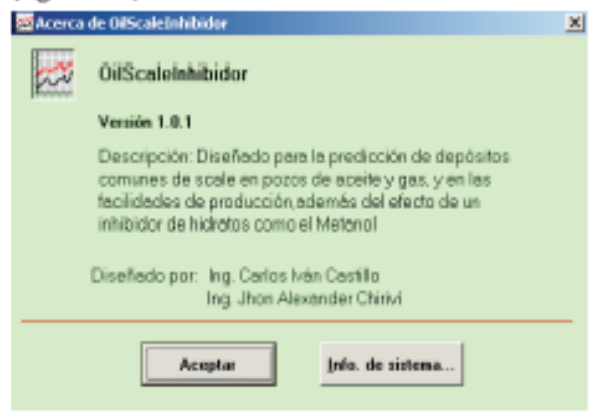

Figura 14. Menú "Acerca de..."

\section{Metodología}

Validación Con Artículos Técnicos (SPE): El proceso de validación de la herramienta de cómputo mediante la información obtenida de artículos técnicos, se llevó a cabo revisando artículos publicados por el grupo de investigación de la Universidad de Rice en los cuales se estudia el efecto de adicionar inhibidor de hidratos (metanol) que generan la formación de depositaciones inorgánicas. Se seleccionaron 2 de los 9 articulos encontrados en la base de datos SPE, ya que estos contenian toda la información necesaria para validar la herramienta de cómputo. Los artículos técnicos seleccionados fueron: SPE 80255 y SPE 100522 .

El artículo técnico SPE 80255 trata sobre el estudio del efecto que se genera al adicionar inhibidores de hidratos de gas causando la formación de depositaciones inorgánicas en un yacimiento petrolifero. En el artículo se enuncia el desarrollo de un modelo de actividad presentado para modelar el efecto de metanol en el equilibrio del solubilidad de calcita, barita, yeso, celestita y halita en una solución gas/ metanol/agua/sal.

El modelo utiliza la teoría de Pitzer para modelar el efecto de la sal y la ecuación de Born para modelar el efecto de metanol. Estos modelamientos se han adicionado a la herramienta de cómputo "OilScaleInhibidor" desarrollado por el grupo de investigación de comportamiento de fases COFA de la Universidad Surcolombiana, en cuyas predicciones se observa que los problemas de depositaciones inorgánicas pueden empeorarse con porcentajes de adición tan pequerios como 5\% (V/V) de metanol, en sistemas característicos de producción de aceite y de gas.

Con los datos recopilados del artículo técnico 80255 se procede a incorporarlos en la herramienta de cómputo OilScalelnhibidor con la cual se obtuvo el valor de los coeficientes de actividad debidos al efecto que se produce al adicionar metanol para la inhibición de hidratos de gas. 
Una vez analizados estos datos, se procedió a ingresarlos a la herramienta de cómputo OilscaleInhibidor para de este modo comparar resultados y conocer la eficacia en la predicción de depositaciones inorgánicas y lo referente a la adición de metanol del programa de cómputo disenáado. Desarrollados los cálculos, el programa predijo los mismos depósitos inorgánicos de los que hablaba el artículo técnico.

\section{Do Resultados}

- Se aplicó la teoría electrolítica de Pitzer para el modelamiento del efecto de la sal y la ecuación de Born para el modelamiento del efecto del alcohol (metanol) para la programación de la herramienta de cómputo "Oilscalelnhibidor".

- La teoría electrolítica de Pitzer, es la teoría que más se aproxima a datos reales en los cálculos de los coeficientes de actividad. Esto se debe a que dicha teoria tiene en cuenta fuerzas de interacción iónica que otros modelos excluyen, tales como, las fuerzas de interacción iónica de corto y largo alcance.

- La herramienta de computo OtIScalelnhibidor, posee una variada interfaz gráfica que facilita el análisis de los resultados, posibilitando al usuario controlar de una forma más versátil, el efecto causado al adicionar inhibidor de hidratos en la formación de depositaciones inorgánicas.

- Realizada la validación de la herramienta de computo OilScalelnhibidor, se observó que cuenta con una buena precisión en los resultados obtenidos. De acuerdo con lo anterior se llegó a las siguiente conclusión:

"Se logró una buena aproximación en el proceso de validación mediante otros programas, a pesar de no tener datos completos. El programa OilScale y el programa ScaleSoftPitzer, son los dos únicos programas de modelamiento termodinámico para la predicción de depositaciones inorgánicas y el efecto causado por el metanol en la formación de depositaciones inorgánicas que se utilizaron para realizar la validación del programa. Como era de esperarse los valores de índice de saturación obtenidos con Oliscale, son muy similares a los valores del ScaleSoftPitzer (con un error promedio absoluto de $8.5 \%$ )".

\section{》O BIBLIOGRAFIA}

1. Kan, Amy T., Gongmin, Watson, Malene A., Mason B., and Tomson. "Effect of hydrate inhibitors on oilfield scale formation and inhibition". SPE 74657. SPE Publications. 2002.

2. Marshal Patricio. "Hidratos de Metano". Petrotecnia 2002.

3. Collet T., Lewis R, Takashi and Uchida. "El creciente interés en los hidratos de gas". Schlumberger. Oilfield Review. Otońo de 2000 .

4. Chen, Dong Songand Zhan. "Prevention and control of gas hydrates for foam combination flooding". SPE 77875. October 2002.

5. McCain William. "The properties of petroleum fluids". Pág 474483. 1993

6. Patton, Charles. Oilfield Water Systems. Campbell Petroleum Series. Oklahoma. 1981.

7. Brandt W., Dang AS., Magne E., Crowley D., Houston K., Rennie A., Hodder M., Stringer R., Juiniti R., Ohara S., and Rushton S. "Deepening the search for offshore hydrocarbons" . Schlumberger. Olffield Review 10. Pág. 2-21

8. Barker JW., and Gomez RD. "Formation of Hydrates During Deepwater Drilling Operations" SPE 16130. 1989.

9. Tohidi B., Danesh A., Burgass RW., and Todd AC. "Effect of heavy hydrate formers on the hydrate free zone of real reservoir fluids" SPE 35568. 1996.

10. AlThubaiti , Kan, Fu, and Tomson. "Scale formation and prevention in the presence 
COFA A Modelamiento termodinámico de los inhibidores de hidratos de gas (metanol)

of hydrate inhibitors". SPE 80255. SPE Publications. 2003.

11. Fu, Tomson, Kan, Shipley $\mathrm{H}$ and Shen D. "Effect of hydrate inhibitors on calcite, sulfates, and halite scale formation". SPE 100522. SPE Publications. 2006.

12. Oddo and Tomson. "A new saturation index equation to predict calcite formation in gas an oil production". SPE 22056. SPE Publications. Octubre 29 de 1990.

13. Prestwich, Rogers, Salimi, Tomson, Varughese, and Waggett. "Use of inhibitors for scale control in brine-producing gas and oil wells". SPE 15457. Presentado en 61a SPE confer encia y exhibición técnica anual. Nueva Orleáns. Octubre 5-8 de 1986.

14. Oddo and Tomson. "Simplified calculation of $\mathrm{CaCO} 3$ saturation at high temperatures and pressures in brine solutions". SPE 10352.

15. www.exp.uji.es/asignatura/obtener. php?letra-N\&.
16. Badillo Carlos y Useche Yina. "Modelamiento termodinámico para la predicción de depositaciones inorgánicas en sistemas de hidrocarburos". Universidad Surcolombiana. 2005.

17. Bonilla Fernando et al. Estado del Arte de las Depositaciones Inorgánicas. Convenio USCO-ICP-ECOPETROL No. 009. Neiva. 2003.

18. Kan, X. Wu, G. Fu, M.B. Tomson, Rice U., Brine Chemistry Consortium." Validation of Scale Prediction Algorithms at Oiffield Conditions". Paper SPE 93264-MS. SPE Publications. 2005.

19. Prausnitz, J. M., Lichtenthaler, R. N. \& De Azevedo, E. "Termodinámica Molecular de los Equilibrios de Fases". 3Ş Edición. Prentice Hall. 2000.)

20. Reid, R., Prausnitz, J. \& Poling, B. "The Properties of Gases and Liquids". 4th Edition. Singapore. McGraw Hill. 1987. 